\title{
Moral dilemma: To operate or not to operate
}

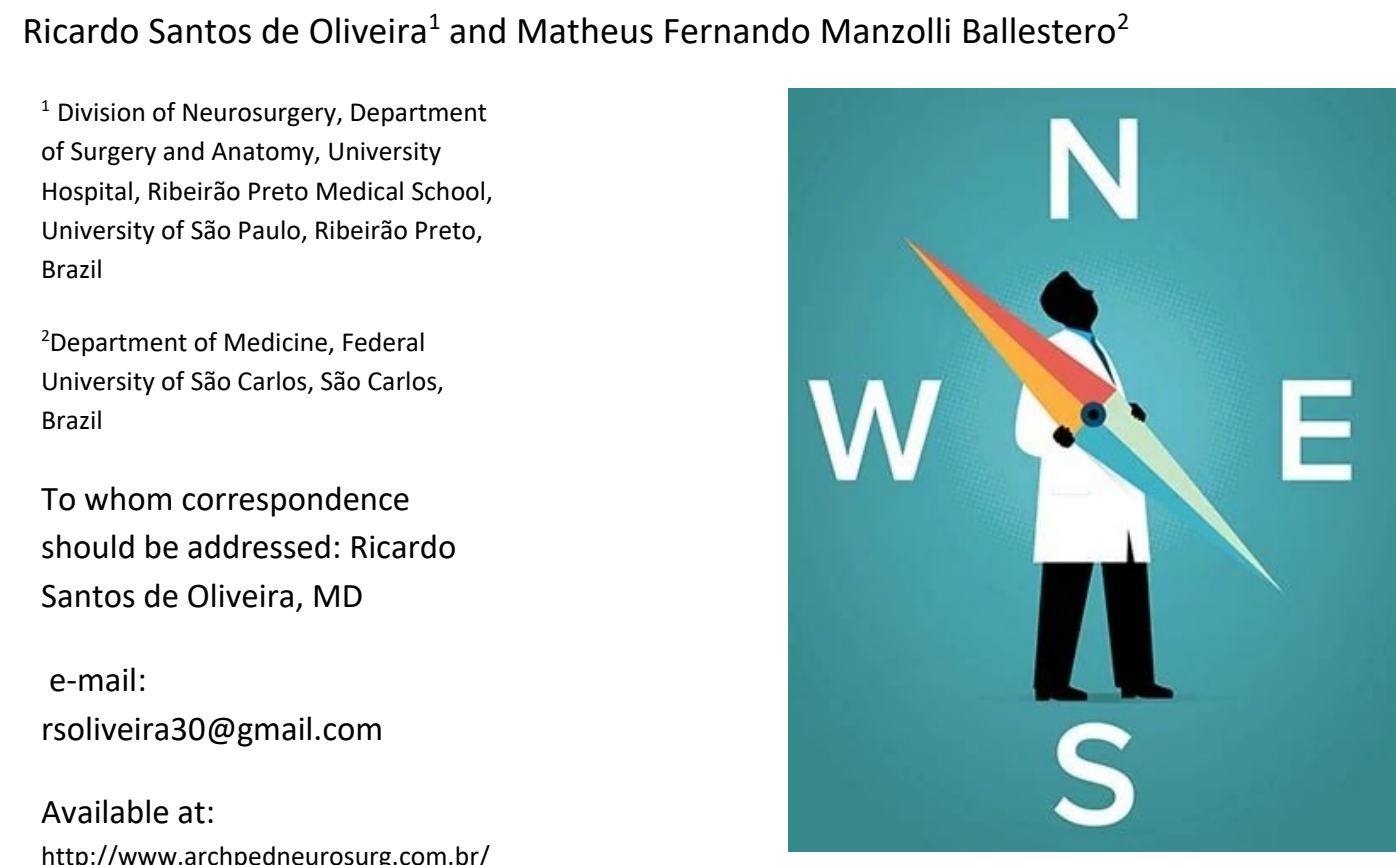

Keyworks: Ethics, Morality, Medico-legal
The practice of surgery is based on the technical capabilities of the surgeon (techne), their knowledge (episteme) and their capacity of judgment (phronesis). Surgeons face situations that call into question moral choices and face ethical difficulties in their daily practice

The routine of being called to evaluate clinical cases in the hospital emergency in the middle of the night is part of the neurosurgeon's life.

They are head trauma, spinal cord trauma, VP shunt dysfunction, cerebral hemorrhage, hydrocephalus, among others. And, we often run into a moral dilemma. To operate or not to operate in situations of extreme gravity and very bad neurological conditions.

The patient and families did not seem to understand what was happening and could neither assent to nor refuse the surgery I was recommending. Yet just hours earlier, he had been his normal self.
Family members are in a desperate situation, and in these moments making a decision involving the life of the loved one is something painful and extremely difficult.

I took him to surgery. The craniotomy went well and he seemed to recover smoothly. But my patient made little improvement over the next two days.

A prolonged ICU stay, tracheostomy, pneumonia, endless blood exams and little neurological evolution. That person will never be the same again.

It is difficult to predict the outcome of this surgery, so the choice to operate or not to operate often generates moral distress in the doctor who makes it. A knowledgeable family who is available to speak on the patient's behalf, or someone with a clearly articulated advance directive, helps guide us. But all too often, patients arrive by ambulance alone or families face these moments in crisis, contemplating death or disability in their family member as if for the first time. 


\section{Moral dilemma: To operate or not to operate}

I go with my gut when deciding whether or not to operate, but always, in the back of my mind, the circumstances stir up doubt. What if I'm wrong? Is it in the patient's best interest to put him through surgery if the chance of meaningful recovery is vanishingly small?

We can also take this discussion also in progressive oncological cases or in severe congenital malformations.

In two articles, "Guidelines for deciding care of critically ill or dying patients," in Pediatrics in 1979, and "Moral and ethical dilemma in the special care nursery" in New Engl J Med in 1973, R. S. Duff classified cases as A, B, or C, depending on how to treat them Class $A$ cases should be treated as much as possible, class B should be treated selectively, and in class $C$ cases, all treatment should be withdrawn $(1,2)$

To determine whether a disease is incurable or not, it is critical to diagnose the condition as accurately as possible and evaluate the outcome. The evaluation of the outcome may change as improvements in medicine take place.

For example, in our field, myelomeningocele (MMC) is the most common malformation. In 1974, an article by J. Lorber, "Selective treatment of MMC," was published in Pediatrics (3). This report is the famous Lorber's adverse criteria, which indicate that cases with high-level MMC, deformation of the spine, severe hydrocephalus, and associated anomalies are inoperable. However, as treatments have improved, these adverse criteria have very rarely been used since the 1990s. On the other hand, improvements in prenatal diagnosis have also led to an increased percentage of termination of pregnancy cases in some countries (4).

In some regions, termination of pregnancy (TOP) for MMC and other congenital malformations are still strictly prohibited due to religious or political reasons (5). And, recently papers showed that fetal surgery for $\mathrm{MMC}$ seems to reduce the incidence of post-natal hydrocephalus. So, there is a constant evolution in the field of medicine. Absolute truths are often put to the test in academia.

The judgment of these cases, involving moral dilemmas, is far from being equated. Notably, the opinion of the review board of the ethical committee was not unanimous.

Even within the same medical environment, neurosurgeons, pediatricians, neonatologists, neurologists, intensivists, have different perspectives, views, and opinions. In some cases, "medico-legal" surgery could be necessary to manage the situation and reducing the pressure under the neurosurgeon.

The core of surgical ethics is the surgeon-patient relationship and the surgeon's responsibility to advance and protect the wellbeing of the patient. It emerged to examine problems specific to surgeons. These problems are closely linked to the development of science and technology.

Moreover, the medical and ethical landscape is constantly shifting, and medical opinions and laws will change over time, according to what the majority of people believe to be true.

The Hippocratic principle "primum non nocere" should be always respect and when deciding on 'why to treat', ethics provides us with four principles: beneficence, nonmaleficence, respect for patient autonomy, and justice (6).

According to the Statement of Ethics in Neurosurgery published by World Federation of Neurological Surgeons (WFNS) in the statement \#5 "We should perform surgery when we believe the patient will substantially benefit, and when the level of risk for complications is acceptable to the patient and family members" and \#84 "When patients are unconscious or medically incompetent, and in the absence of any advance directives from the patient declining such intervention, we are responsible to perform procedures that are lifesaving and prevent severe or permanent disability. Depending on circumstances, and on national law and custom, treatment decisions may be made after discussions with relatives, consideration by a hospital ethics committee, or legal advisement" (7).

Surgeons should be skilled in the art and science of surgery and be trustworthy from an ethical and moral standpoint.

\section{REFERENCES}

1. Duff RS: Guidelines for deciding care of critically ill or dying patients. Pediatrics 64: 17-23, 1979

2. Duff RS, Campbell AG: Moral and ethical dilemmas in the special-care nursery. N Engl J Med 289: 890-894, 1973

3. Lorber J: Selective treatment of myelomeningocele: To treat or not to treat? Pediatrics 53: 307-308, 1974

4. Yamasaki M. Life and Medical Ethics in Pediatric Neurosurgery. Neurol Med Chir (Tokyo). 2017 Feb 15;57(2):101-105. doi: 10.2176/nmc.ra.2016-0122. 